\title{
Enhancing the oral bioavailability of natural astaxanthin using plant- based micro- and nano-encapsulation materials: Results of an in vitro evaluation and a crossover study in humans
}

Yarden Abuhassira-Cohen ${ }^{\mathrm{a}}$, Ravit Edelman ${ }^{\mathrm{a}}$, Randa Abbas ${ }^{\mathrm{b}}$, Daniel Kurnik ${ }^{\mathrm{c}}$, Rand Shibel ${ }^{\mathrm{a}}$ and Yoav

\author{
D. Livney ${ }^{\mathrm{a} *}$
}

${ }^{a}$ The Laboratory of Biopolymers for Food and Health, Department of Biotechnology and Food

Engineering, Technion - Israel Institute of Technology, Haifa 3200000, Israel

${ }^{\mathrm{b}}$ Enzymotec-Frutarom Ltd, Israel.

${ }^{\mathrm{c}}$ Clinical Pharmacology Unit, Rambam Health Care Campus, and Rappaport Faculty of Medicine, Technion - Israel Institute of Technology, Haifa, Israel.

Submitted: May 21, 2020

Accepted: June 10, 2020

Published: August 31, 2020

\section{Graphical abstract}

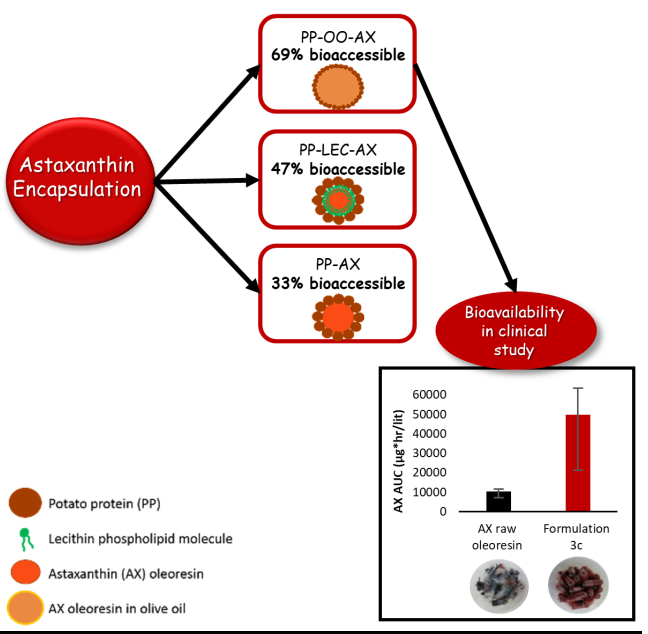

We developed a potato protein-based delivery-system for lipophilic bioactives, using astaxanthin as a model. The oral bioavailability of astaxanthin in humans was approx. 5 times higher than that of astaxanthin oleoresin using this delivery system.

\section{Abstract}

Astaxanthin (AX) is a red xanthophyll carotenoid found mainly in algae (notably Haematococcus Pluvialis microalga) and marine animals. $\mathrm{AX}$ is a stronger antioxidant than vitamin $\mathrm{E}$ and $\beta$-carotene but has very low oral bioavailability. The purpose of this study was to develop a potato protein (PP)based delivery system for increasing oral bioavailability of lipophilic bioactives (nutraceuticals and drugs), using AX as a model, and to evaluate the system in vitro and in vivo in a crossover clinical study in human volunteers. Three different formulations were prepared, encapsulating AX oleoresin (AXO) with (1) PP only, (2) PP+lecithin (LEC), and (3) PP+olive oil (OO). The average particle diameters after preparation were $0.29,0.29$, and $1.76 \mu \mathrm{m}$, and after freeze-drying and reconstitution $0.17,0.07$, and $6.93 \mu \mathrm{m}$, respectively. In vitro bioaccessibility was 33,47 , and $69 \%$, respectively, versus $16 \%$ only for the raw AXO. In a randomized, double-blind, crossover study in human subjects, the PP-OO-AX formulation had a 4.8-fold higher median plasma $\mathrm{AX}$ area under the concentration-over time curve (AUC; $\mathrm{P}<0.001$ ) compared to the raw AXO formulation. In conclusion, a non-allergenic, vegan, PPbased delivery system made of "all-natural ingredients" offers a great promise for increasing oral bioavailability of lipophilic bioactives such as AX, for the enrichment of food and for dietary supplements, or oral delivery of lipophilic drugs.

Keywords: Astaxanthin; Bioavailability; Encapsulation; Potato protein; Olive oil

*Corresponding author: Prof. Yoav D. Livney: livney@technion.ac.i1 


\section{Abbreviations:}

$\begin{array}{ll}\text { AX } & \text { astaxanthin } \\ \text { AXO } & \text { AX oleoresin } \\ \text { AUC } & \begin{array}{l}\text { area under the curve } \\ \text { biopharmaceutics classification } \\ \text { BCS }\end{array} \\ & \text { gystem } \\ \text { GRAS } & \text { lecithin } \\ \text { LEC } & \text { olive oil } \\ \text { OO } & \text { potato protein } \\ \text { PP } & \text { simulated bile fluid } \\ \text { SBF } & \text { simulated gastric fluid } \\ \text { SGF } & \text { simulated intestinal fluid } \\ \text { SIF } & \text { Rationale and Purpose }\end{array}$

Natural AX represents a highly potent but expensive antioxidant with low oral bioavailability. There is an unmet need to protect, solubilize, and enhance the oral bioavailability of such compounds, using only safe, natural, non-allergenic, and inexpensive ingredients. This study aimed to develop a PP-based delivery system for increasing the oral bioavailability of lipophilic bioactives, using AX as a model. This delivery system offers a great promise for enhancing the oral bioavailability of different lipophilic bioactives and the enrichment of food for dietary supplements and for oral delivery of drugs.

\section{Introduction}

Astaxanthin (AX) $\left(3,3^{\prime}\right.$-dihydroxy- $\beta$ - $\beta$-carotene-4,4'-dione; Figure 1) is a xanthophyll carotenoid [1], [2] found mainly in algae and marine animals [3] (e.g., salmon, trout, and lobster, and even flamingo), conferring them their characteristic red-orange color. It can be synthesized only by a few microorganisms, notably the green microalga Haematococcus Pluvialis, which can accumulate it up to $6 \%$ of its dry weight [4].

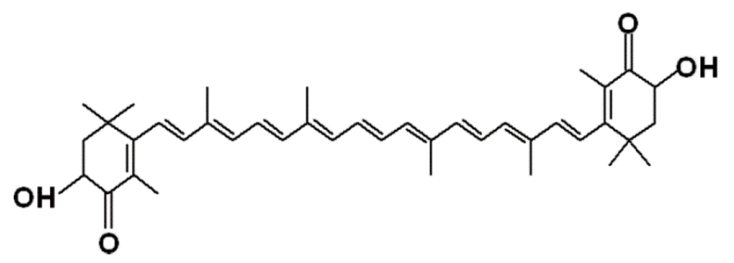

Figure 1 - Planar structure of Astaxanthin [1]

AX is a potent antioxidant due to its many conjugated double bonds. It exhibits stronger antioxidant activity than vitamin $\mathrm{E}$ and $\beta$-carotene, attributed to its strong reactive-oxygenspecies and free radical scavenging activity [5], [6]. Moreover, AX can activate different cellular antioxidant defense systems for skin protection by upregulating stress-sensitive transcription factors and consequently, also oxidative stress-responsive enzymes [7]. Due to its antioxidant properties, AX has many purported health benefits, such as anti-inflammatory, anticancer, cardio- and skin-protective activities [6]. Furthermore, due to its unique structure, it becomes embedded across the cell membrane [1] hence may be highly protective against membrane oxidation.

$\mathrm{AX}$ is known to be safe and has been approved as a food colorant by the USFDA [1]. A major limitation of $\mathrm{AX}$ is its low bioavailability. According to the biopharmaceutics classification system (BCS), the bioavailability is mainly determined by aqueous solubility and by intestinal permeability [8]. Pure AX has almost no solubility in aqueous systems $\left(7.9 \times 10^{-}\right.$ ${ }^{10} \mathrm{mg} / \mathrm{L}$ at $25^{\circ} \mathrm{C}$ [9]) and tends to be crystalline in room or body temperature (its melting point is $182-183^{\circ} \mathrm{C}$ [9]). Crystalline materials are known to have low permeability via the intestinal mucosa [10]. Hence, AX would most appropriately be classified as BCS class IV (low solubility and low permeability). This may explain its low absorption rate and low bioavailability [11].

Potato proteins (PP) are produced from a widely available, inexpensive raw material, and are a by-product of potato starch production. They are considered "generally recognized as safe" (GRAS) and non-allergenic, natural, and applicable in vegetarian and vegan products [12]. PPs have high nutritional value [13], and are commonly divided into three fractions: patatin, protease inhibitors, and other high molecular weight proteins. Different PP fractions are water-soluble at different $\mathrm{pH}$ ranges [14]. Furthermore, PPs are amphiphilic so that they can be particularly useful for the encapsulation of hydrophobic compounds with low water-solubility [12].

The delivery of bioactives into the human body is highly affected by particle size. Thus micro- and, even more so, nano-encapsulation of bioactive components with poor solubility in 
aqueous solutions can improve their dispersibility in water, and therefore also their bioavailability and bioactivity [15], [16].

Protein-based nano-encapsulation technologies have been found effective in protecting the encapsulated bioactive during thermal treatment [12], [17], exposure to UV [18] or visible light [12], [19], low $\mathrm{pH}$ [20], during storage [12], [18], [19], and during digestion [19], [21]. High bioavailability of hydrophobic nutraceuticals, (e.g., vitamin D), nano-encapsulated in protein nanoparticles (e.g. casein micelles), was clinically demonstrated in humans [22], [23]. Bioavailability of vitamin D was found to be comparably high in protein nanoparticles as in milk fat in a clinical study [22].

Several approaches for increasing AX dispersibility and bioavailability have been suggested thus far. In vivo (rat model), bioavailability of AX was improved when ingested as $\mathrm{O} / \mathrm{W}$ emulsion. Better AX absorption was found with $\mathrm{OO}$ than with corn oil, indicating the oil type is also important [24]. Another study compared AX bioavailability in sesame oil versus in gelatin. Surprisingly, the bioavailability of AX in gelatin (a protein) was higher than that in oil [25]. AX microencapsulation with soy LEC and gum Arabic or whey protein was studied using spray drying, while thermal- and $\mathrm{pH}$ stability was tested. Whey protein was found preferable, considering powder color and high antioxidant stability of the AX after encapsulation [26].

Herein, we have developed a novel, foodgrade delivery system to enhance the bioavailability of AX. The microencapsulated AX powder was filled into water-soluble, closable capsules and tested in a crossover study on human volunteers to determine its relative bioavailability and toward its future application in food or dietary supplements.

\section{Experimental design}

The aim was to develop a delivery system to enhance the bioavailability of AX, using PP as a shell-material and combine LEC or OO as absorption enhancers, because carotenoids are thought to be absorbed via the lipid absorption route; hence the presence of fatty acids (either in a triglyceride or a phospholipid) would facilitate this route. To choose the best formulation for this purpose we analyzed the different formulations and evaluated their bioaccessibility in vitro. First, we prepared samples with different ratios of PP, AX, LEC, and OO. We performed particle size analysis and microscope evaluation to choose the best ratio for each formulation. Samples with the best ratio underwent simulated digestion to evaluate their in vitro bioaccessibility. The best-performing formulation was chosen and was tested in a clinical trial to evaluate the in vivo relative bioavailability and the efficacy of our delivery system, comparing microencapsulated with raw $\mathrm{AXO}$ at the same AX dose. Briefly, after a night fast, a blood sample was taken from each subject $(\mathrm{t}=0)$. Then, they consumed a standardized "meal" (yogurt). After 15 minutes, they ingested the AX capsules (external capsule made of pullulan- a natural water-soluble polysaccharide, for release in the stomach), microencapsulated or raw $\mathrm{AXO}$, depending on a predetermined random sequence. Blood samples were collected at pre-specified time points for the analysis of plasma AX concentrations. After a washout period of 1 week (given that the reported plasma half life time of AX is about 15 hours [27], one week is well above the 5 halflife times required for a washout period), for the second ingestion, the same procedure was followed, with the subject receiving the other AX formulation as determined by the random sequence. Based on a previous clinical study [27], a sample size of 10 subjects was calculated to give $90 \%$ power and show statistically significant differences in AX exposure between the 2 formulations $(\alpha=0.05)$. Assuming three dropouts, we recruited 13 subjects, and all completed the study (See further details in the methods section).

\section{Materials and Methods}

\section{Materials}

PP isolate (Solanic 200, AVEBE) was provided by Nutrafur (Murcia, Spain). Natural AXO (10\% Astaxanthin) and tapioca maltodextrin were provided by Frutarom Ltd, (Herzeliya, Israel). Sunflower LEC (powder) was purchased from Texturot (Israel). Refined OO was provided by S.M. Natural Ingredients (Rishon leZion, Israel). pepsin (P7000), trypsin (T0303), chymotrypsin (C4129), lipase (L3126), taurocholic acid sodium salt hydrate (T4009), sodium glycodeoxycholate (G9910), and Pefabloc ${ }^{\circledR}$ SC (PEFBSC-RO) were purchased from Sigma Aldrich (Rehovot, Israel). 


\section{Stock solution preparation}

PP was dissolved in phosphate buffer $(\mathrm{pH}=$ 7) at $37^{\circ} \mathrm{C}$ for 45 minutes (PP concentration 1 $\mathrm{mM}$ ). After cooling to room temperature, the solution was centrifuged to precipitate insoluble matter (3000 rpm, $5 \mathrm{~min}$ ). The supernatant was collected and filtered by vacuum filtration ( $0.45 \mu \mathrm{m}$ filter). The final protein concentration was determined by a spectrophotometer. AXO was dissolved in absolute ethanol at $50{ }^{\circ} \mathrm{C}$ for 30 minutes to obtain an ethanolic AX stock solution $(10 \mathrm{mM})$. Sunflower LEC was dissolved in $20 \mathrm{~mL}$ of absolute ethanol for 2 hours (LEC concentration $27 \mathrm{mM}$ ). The solution was centrifuged for 10 minutes at $5000 \mathrm{rpm}$. The supernatant containing the ethanol-soluble fraction of LEC was collected. The final concentration was determined gravimetrically by evaporating the ethanol from the supernatant and was found to be $13.5 \mathrm{mM}$.

Encapsulation of $A X$ within $P P$, with or without lecithin or olive oil.

\section{AX-PP nanoparticles (NPs) formation}

An ethanolic stock solution of AXO was added dropwise into PP solution, during vortexing, to obtain 2 different molar ratios. The molar ratios obtained were $1: 1$ and 2:1 (AX:PP). $\mathrm{AX}$ concentration was $0.5 \mathrm{mM}$ in all samples.

\section{$A X+L E C-P P$ NPs formation}

A pre-mix of the AX stock solution with the ethanol-soluble fraction of sunflower LEC was prepared (equal concentrations of AX and LEC: $5 \mathrm{mM}$ in absolute ethanol). The pre-mix was added dropwise into PP solution, during vortexing, to obtain different molar ratios $(\mathrm{AX}+\mathrm{LEC}$ in excess). The molar ratios obtained were 1:1:1 and 2:2:1 (AX:LEC:PP). AX concentration was $0.5 \mathrm{mM}$ in all samples.

\section{$\mathrm{AX}+\mathrm{OO}-\mathrm{PP}$ emulsion preparation}

A solution of AXO and refined OO $(1: 3 \mathrm{w} / \mathrm{w}$ AXO:OO) was shaken at $50^{\circ} \mathrm{C}$ for 30 minutes until it was homogenous. PP solution (in phosphate buffer, $\mathrm{pH}=7$ ) was added to the $\mathrm{AX}-\mathrm{OO}$ solution. Two different proportions were tested: 1:3:4 w/w/w AXO:OO:PP in the final solution (4\% oil) or $0.5: 1.5: 4 \mathrm{w} / \mathrm{w} / \mathrm{w}$ AXO:OO:PP in the final solution ( $2 \%$ oil). Then, the mixture was pre-homogenized using a desktop homogenizer (30,000 rpm, 5 minutes). The pre-emulsion obtained was homogenized using a high-pressure homogenizer (Emulsiflex C3, Avesin, 950 bar, up to 4 passes).

\section{System characterization}

\section{Particle size distribution}

Particle size distribution of AX-PP and AX+LEC-PP samples was determined using a NICOMP DLS analyzer. Particle size distribution of $\mathrm{AX}+\mathrm{OO}-\mathrm{PP}$ emulsion was determined by laser diffraction using a Malvern Mastersizer 3000. Statistical analysis of the results was performed using Microsoft Excel 2013. The samples were tested in duplicate.

\section{Light microscopy}

Crystal morphology and particle size were studied by light microscopy, using an Olympus DP71 digital camera connected to an Olympus BX51 light microscope, operated in a brightfield optical mode or in polarized light optics.

Examining freeze-drying and powder re-

\section{constitution}

Samples were freeze-dried using a Labconco benchtop Freeze-Dryer. Then, the samples were reconstituted to the initial volume with distilled water and kept at $-20^{\circ} \mathrm{C}$ until simulated digestion or particle size analysis were performed.

Simulated digestion of the powders and evaluation of $A X$ in vitro bioaccessibility

Simulated gastric and intestinal digestion was based on the protocol described by Minekus et al. [28]. Briefly, four parts of simulated gastric fluid (SGF), one part of pepsin dissolved in SGF (final concentration in the digestion mixture: 2000 units $/ \mathrm{mL}$ ) and $\mathrm{CaCl}_{2}$ (final concentration in the digestion mixture: $0.075 \mathrm{mM}$ ) were added to five parts of a reconstituted sample ( $375 \mu \mathrm{L}$ at $0.5 \mathrm{mM} \mathrm{AX})$ and the $\mathrm{pH}$ was adjusted to 3 . After 2 hours, one part of the gastric digesta was mixed with one part of simulated intestinal fluid (SIF) containing duodenal enzymes and bile salts, and the $\mathrm{pH}$ was adjusted to 7.0. Each of the following enzymes was dissolved in SIF before adding to the digesta: trypsin (final concentration in the digestion mixture: 100 units $/ \mathrm{mL})$, chymotrypsin (25 units $/ \mathrm{mL}$ ), and lipase (2000 units $/ \mathrm{mL}$ ). The bile salts, sodium glycocholate- and taurocholic acid were dissolved in simulated bile fluid (SBF) to achieve a final concentration of 10 $\mathrm{mM}$ bile salts ( $5 \mathrm{mM}$ each) in the digestion mixture. The digestion was stopped 2 hours after 
initiating intestinal conditions (4 hours from the start of the gastric stage) using Pefabloc. To evaluate the bioaccessibility of $\mathrm{AX}$, the digested samples underwent ultra-centrifugation $\left(15,000 \mathrm{rpm}, 30\right.$ minutes, $\left.20^{\circ} \mathrm{C}\right)$, the supernatant was collected, and its AX content (the bioaccessible fraction) was determined by extraction and reverse-phase HPLC (RP HPLC). Extraction by phase separation was achieved by adding dichloromethane and methanol (1:3 $\mathrm{v} / \mathrm{v})$, followed by 20 seconds of vortexing. Two $\mathrm{mL}$ of dichloromethane were then added to each test tube, and it was vortexed for $1 \mathrm{~min}$. The samples were then centrifuged for $10 \mathrm{~min}$ at $1,500 \mathrm{G}$ at $4{ }^{\circ} \mathrm{C}$. The bottom layer of dichloromethane containing AX was removed, and the steps of the addition of $2 \mathrm{~mL}$ of dichloromethane were repeated twice more. Dichloromethane was evaporated under a flow of nitrogen, and the extract was reconstituted in a known volume of ethanol.

\section{Scale-up}

After choosing the most bioaccessible formulation, process changes were made to produce the capsules at a larger scale for the bioavailability study in humans. AX+OO-PP emulsion was made as described above with slight changes. First, the $4 \%$ oil formulation was made using a high-pressure homogenizer (Nano DeBEE, 28,000 psi, 2 passes). To increase the percentage of $\mathrm{AX}$ in the final powder, the proportions in the formulation were changed to $1: 2: 3 \mathrm{w} / \mathrm{w} / \mathrm{w}$ AXO:OO:PP in the final solution, and tapioca maltodextrin was added $(1 / 20 \mathrm{w} / \mathrm{w}$ of the protein amount) as a cryoprotectant. The emulsions were freeze-dried, and particle size distribution and bioaccessibility were evaluated as described above. Finally, the dried powder was filled into pullulan capsules. The dose was $15 \mathrm{mg}$ AX /4 capsules.

\section{Bioavailability study in human volun-}

\section{teers}

The relative oral bioavailability of the most bioaccessible formulation was compared to that of the raw AXO in a crossover study in human volunteers, complying with the World Medical Association Declaration of Helsinki. The Rambam Health Care Campus Institutional Ethical Committee approved the study protocol (RMB0048-18; Ministry of Health approval number 20185271), and all participants gave written in- formed consent. We explored the relative bioavailability of $A X$ in humans in 2 different formulations: (A) Raw AXO and (B) Microencapsulated AX (1\%:2\%:3\% (AXO:OO:PP, \%w/v ratio) $+0.15 \%$ maltodextrin). The two formulations were compared in a randomized, singledose, double-blind, two-period, two-sequence, crossover study performed at the Rambam Health Care Campus. Subjects were eligible if they were aged 18 to 26 years and healthy, as determined by a normal physical examination, normal electrocardiogram, and normal routine laboratory examinations (biochemistry and complete blood count). Subjects with any active disease, lactose intolerance or food allergies, smoking, excessive alcohol use (over 40 $\mathrm{mL}$ /day), pregnancy or breast-feeding, hyperlipidemia (LDL $>130$, triglycerides $>200$ ), regular medication use, obesity (BMI $\left.>30 \mathrm{~kg} / \mathrm{m}^{2}\right)$, or use of multivitamins or carotenoid supplements during the last month before the study were excluded. After a night fast, in the morning of the first study day, the subjects reported to the study center at the Rambam Health Care Campus, and a fasting blood sample was taken $(\mathrm{t}=0)$. Then, they consumed a standardized 300 gr portion of fat-free yogurt containing $20 \mathrm{~g}$ of sugar. After 15 minutes, they swallowed 4 AX pullulan capsules containing a total of $15 \mathrm{mg}$ of $\mathrm{AX}$ (formulation $\mathrm{A}$ or $\mathrm{B}$, depending on the predetermined random sequence) with $400 \mathrm{~mL}$ of water, and blood samples were collected after 2, 4, 6, 8, 10, 24, 48 and 72 hr. Plasma AX levels were determined by HPLC. After a washout period of 1 week, on the second study day, the same procedure was followed, with the subject receiving the other $\mathrm{AX}$ formulation, as determined by the random sequence.

\section{Dosage information}

The recommended dose of astaxanthin is 2-4 $\mathrm{mg}$ /day [1]. The doses reported in various studies for daily intake range from $0.7-22 \mathrm{mg}$ /day [1]. In previous studies reported in the literature, a single dose of $100 \mathrm{mg}$ [29] and repeated doses of up to $22 \mathrm{mg} /$ day for 14 days [30] were given to healthy volunteers without adverse events. The most similar study reported (only using synthetic surfactants) used $40 \mathrm{mg}$ as a single dose [27]. Herein, we chose to give a dose of $15 \mathrm{mg}$ of $\mathrm{AX}$, single intake, which is well within the safe range for a single dose, and higher than the recommended daily intake, to improve the analytical quantification in the 
plasma. The intended dose in envisioned future products would likely be the recommended daily amount. In each of the two compared formulations, the dose was given in four pullulan capsules. While it may be possible to obtain 4 mg AX daily from the diet (e.g., from 185 grams of salmon per day [1]), most people consume much lower amounts; hence a dietary supplement seems an effective way to provide the recommended dose.

\section{Plasma AX levels kinetic study calcula-}

\section{tions}

Plasma AX concentrations were plotted over time for each subject, and AX formulation. The area under the curve (AUC) of the plasma concentration over time was calculated using the trapezoidal rule. $T_{1 / 2}$ was calculated by equation 1:

$$
\text { Equation } 1 \quad T_{1 / 2}=\frac{\ln 2}{k_{e}}, k_{e}=\frac{\ln C_{1}-\ln C_{2}}{t_{2}-t_{1}}
$$

where the elimination rate constant $\mathrm{k}_{\mathrm{e}}$ was determined from the terminal slope of the semilogarithmic graph of concentration over time, with $C_{x}=$ the concentration at $t_{x}$, the peak concentration $\left(\mathrm{C}_{\max }\right)$, the time to peak concentration $\left(\mathrm{T}_{\max }\right)$, and the lag time, i.e., the time from dosing until an increase in AX concentrations $\left(\mathrm{T}_{\text {lag }}\right)$, were read directly from the graph.

Comparisons between the two treatments were conducted in two ways: on the concentrations determined during the full time-course, and on the summarizing parameters based on the entire time course, such as AUC, $\mathrm{C}_{\max }, \mathrm{T}_{\max }$, $\mathrm{T}_{\text {lag }}$, and $\mathrm{T}_{1 / 2}$.

\section{Statistical methods}

Initial analysis of AUC, $\mathrm{C}_{\max }, \mathrm{T}_{\max }, \mathrm{T}_{\mathrm{lag}}$, and $\mathrm{T}_{1 / 2}$ indicated that a non-parametric statistical analysis would be most suitable. Thus, the Wilcoxon signed-rank test was employed. To examine changes in concentration along the timecourse, a repeated-measures (mixed model)
ANOVA was performed. ANCOVA was considered, using the pre-treatment scores as the covariate, but concentrations at that time were either zero or much lower than at later, posttreatment time-points; thus, ANOVA including those pre-treatment time points was performed. A full factorial model was utilized, with treatment, time, and their interaction serving as fixed effects. Pre-planned, Bonferroni-corrected slice contrasts, comparing concentrations at each time point, were performed. Initial analyses indicated that a logarithmic transformation would be required to alleviate the nonnormality of ANOVA residuals, so to accommodate zero values, the transformation $\log 10$ (AX concentration +1 ) was utilized. Several different alternative covariance structures were evaluated by AICc (Akaike Information Criterion - corrected) criteria, including autoregressive models (to account for possible correlations of concentrations closer to each other in time); none performed better than the conventional compound symmetry covariance structure, which was employed. JMP software was employed in the initial analyses, and for Wilcoxon signed rank tests, and SAS was used for the mixed-model ANOVA (both packages from SAS Institute, Cary, NC, USA).

\section{Results}

Different formulations of AX within PP, with or without lecithin or olive oil, were prepared as described above and were characterized for particle size distribution and reconstitutability, using DLS, or laser diffraction, and light microscopy. As a control for evaluating the effect of the PP on particle properties, the unencapsulated AXO particles were prepared by dissolving AXO in ethanol and adding into the buffer while stirring.

\section{Particle size distribution}

AX was encapsulated within PP, with or without LEC/OO, as described above. The particle size distribution is shown in Figure 2. 

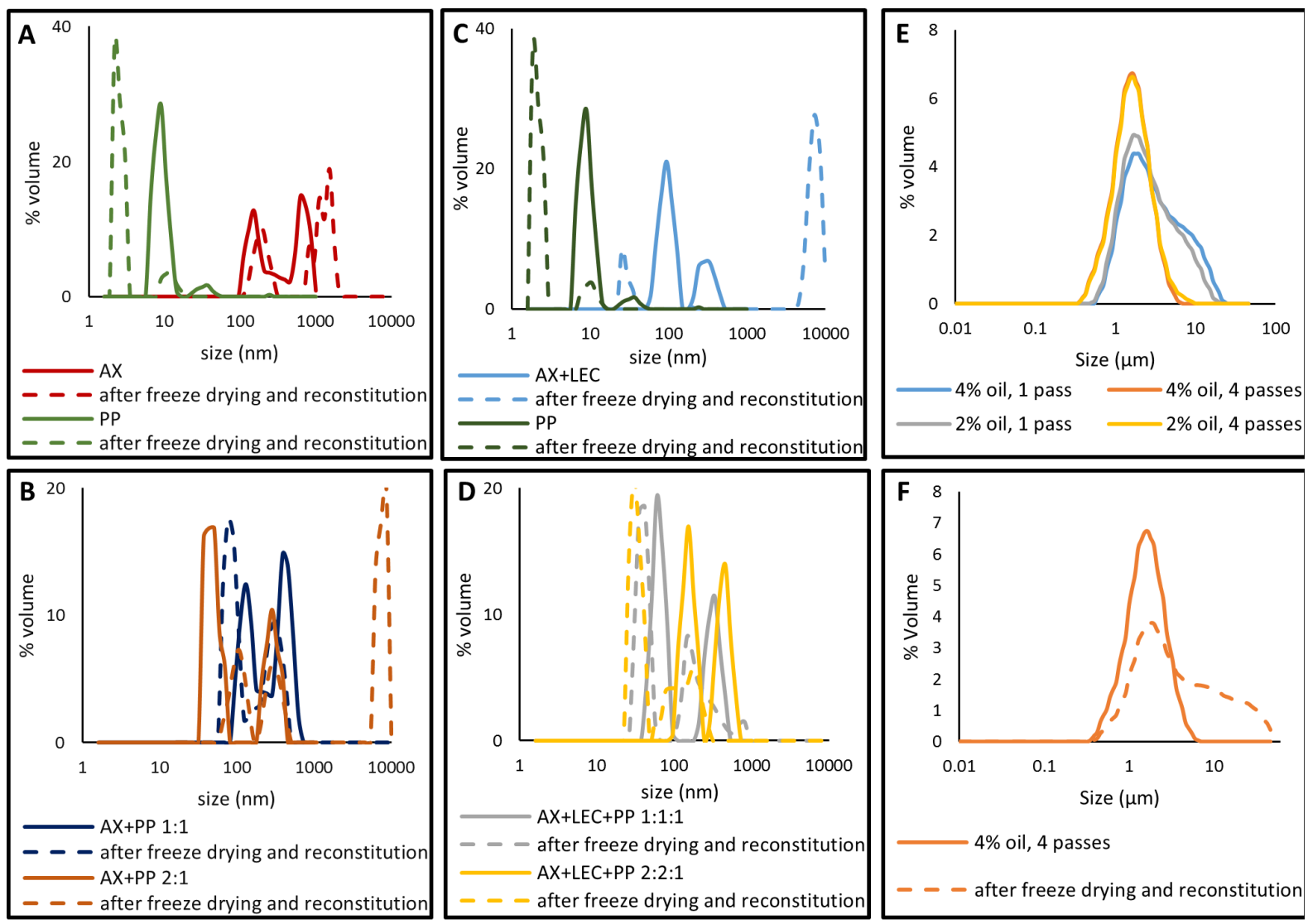

Figure 2 - Particle size distribution of $A X$ in different encapsulations with $P P-(A)$ unencapsulated AXO microdroplets in buffer $(0.5 \mathrm{mM})$ and pure $P P(0.5 \mathrm{mM})$, (B) AX-PP NPs at 1:1 and 2:1 molar ratios (AX:PP); PP concentration was $0.5 \mathrm{mM}$ (C) AX+LEC stock solution $(0.5 \mathrm{mM})$ in buffer and $P P(0.5 \mathrm{mM})$, (D) AX+LEC-PP NPs at 1:1:1 and 2:2:1 molar ratios (AX:LEC:PP); PP concentration was $0.5 \mathrm{mM}$, (E) AX+OO-PP emulsions, $1: 3: 4 \mathrm{w} / \mathrm{w} / \mathrm{w}$ AXO:OO:PP (4\% oil) and 0.5:1.5:4 w/w/w AXO:OO:PP (2\% oil), after 1 or 4 passes in a high pressure homogenizer ( $F)$ AX+OO-PP 4\% oil emulsion, 4 passes in a high pressure homogenizer, before and after freeze-drying and reconstitution. AX = astaxanthin; $A X O=A X$ oleoresin; $L E C=$ lecithin; $N P S=$ nanoparticles; $O O=$ olive oil; $P P=$ potato protein

The average diameter of unencapsulated AXO droplets (Figure 2A) was 423.3 and 771.6 $\mathrm{nm}$, before and after freeze-drying and reconstitution, respectively. For PP alone (Figure 2A), the average particle diameter was 11.1 and 3.1 $\mathrm{nm}$, before and after freeze-drying and reconstitution, respectively. For the AX-PP NPs (Figure $2 \mathrm{~B}$ ), at 1:1 molar ratio (AX:PP) the average particle diameter was 288.3 and $174.4 \mathrm{~nm}$, and at 2:1 molar ratio (AX:PP) the average particle diameter was 134.3 and $3942.9 \mathrm{~nm}$, before and after freeze-drying and reconstitution, respectively. The 1:1 molar ratio showed reduction in particle size compared to unencapsulated AXO. Also, after freeze-drying and reconstitution, the average particle diameter was smaller at $1: 1$ molar ratio, compared to the same ratio before drying, which indicates better reconstitution ability than at 2:1 molar ratio; therefore the 1:1 molar ratio (Formulation 1) was chosen for further study. The average particle diameters of unencapsulated AXO+LEC (Figure 2C) were 156.0 and $6177.0 \mathrm{~nm}$, before and after freezedrying and reconstitution, respectively. For the AX+LEC-PP NPs, at 1:1:1 molar ratio (AX:LEC:PP) (Figure 2D), the average particle diameters were 166.8 and $129.8 \mathrm{~nm}$, and at 2:2:1 molar ratio (AX: LEC:PP), the average particle diameters were 285.2 and $74.8 \mathrm{~nm}$, before and after freeze-drying and reconstitution, respectively. The 1:1:1 and 2:2:1 molar ratios showed a reduction in particle size compared to unencapsulated AXO+LEC. In addition, after freeze-drying and reconstitution, the average particle diameters were smaller in both molar ratios, compared to the same ratio before drying, which indicated good reconstitution ability. The particle size was smaller at 2:2:1 molar ratio compared to that at 1:1:1 molar ratio, and therefore 2:2:1 (Formulation 2) was chosen for the bioaccessibility study. For the AX+OO-PP emulsion, different compositions were tested: 
1:3:4 w/w/w AXO:OO:PP in the final solution (4\% oil), and 0.5:1.5:4 w/w/w AXO:OO:PP in the final solution $(2 \%$ oil $)$, and each one was tested after one or four passes through a highpressure homogenizer. For the $4 \%$ oil (Figure $2 \mathrm{E}$ ), the average particle sizes were 4.2 and 1.8 $\mu \mathrm{m}$, for one and four passes, respectively. For the $2 \%$ oil (Figure $2 \mathrm{~F}$ ), the average particle sizes were 3.4 and $1.8 \mu \mathrm{m}$, for one and four passes, respectively. The $4 \%$ oil, 4 passes through high-pressure homogenization (For- mulation 3), yielded the smallest average particle size and highest AX content, and after freeze-drying, the average particle size was 6.9 $\mu \mathrm{m}$.

\section{Light microscopy}

Each of the chosen formulations was studied by light microscopy. According to the light microscopy images, all three formulations showed smaller particle sizes, compared to unencapsulated AXO (Figure 3).
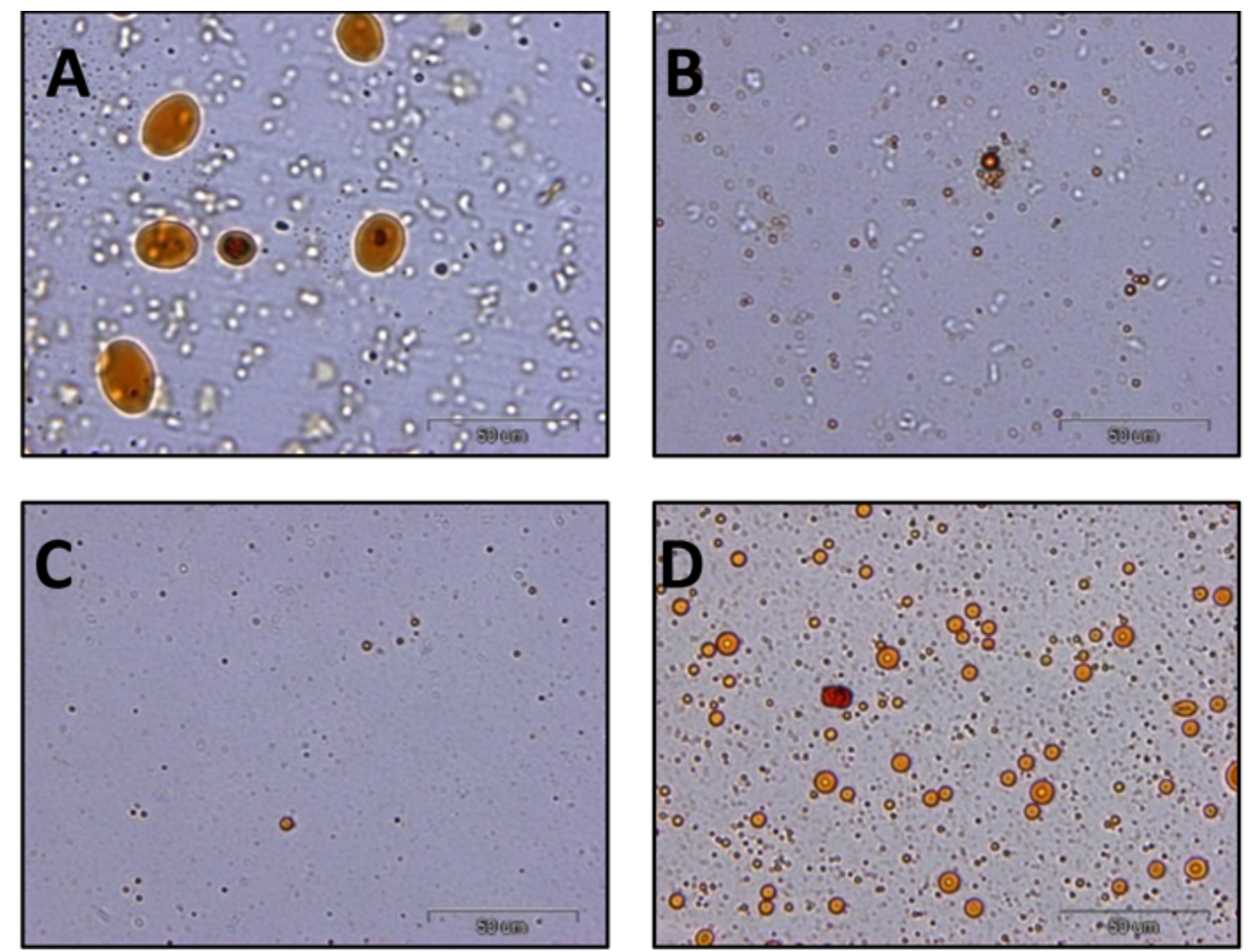

Figure 3 - Light microscope images of (A) unencapsulated AXO in buffer, showing formation of microdroplets; (B) Formulation $1-A X-P P N P$ at 1:1 molar ratio (AX:PP); (C) Formulation $2-A X+L E C-P P$ NPs at 2:2:1 molar ratio (AX:LEC:PP); and (D) Formulation $3-A X+O O-P P$ emulsion at 1\%:3\%:4\% (AXO:OO:PP, \%w/v ratio), after 4 passes through the highpressure homogenizer. $A X=$ astaxanthin; $A X O=$ AX oleoresin; $L E C=$ lecithin; $N P$ s $=$ nanoparticles; $O O=$ olive oil; $P P=$ potato protein.

Formulation 2 (AX+LEC-PP NPs, at 2:2:1 molar ratio) showed the smallest particles of all studied formulations (Figure 3C). These results are in accord with the particle size distributions obtained by the DLS or the Mastersizer.

\section{In vitro bioaccessibility of $A X$}

Each of the chosen formulations underwent simulated gastrointestinal digestion and ultracentrifugation, as described above, to evaluate the in vitro bioaccessibility. AX was quantified in the supernatant by RP-HPLC as described above.
The bioaccessibility of unencapsulated AXO particles was $(16 \pm 3) \%$, and it was $(33 \pm 3) \%$ and $(47 \pm 8) \%$ for Formulations 1 and 2, respectively (Figure 4). The bioaccessibility was calculated as percent of the known $\mathrm{AX}$ concentration in the original sample. Formulation 3 (1:3:4 w/w/w AXO:OO:PP in the final solution) showed the highest bioaccessibility, $(69 \pm 4) \%$, and therefore, this type of formulation, i.e., containing $\mathrm{AX}, \mathrm{OO}$, and $\mathrm{PP}$, was chosen for scaleup and bioavailability study in humans compared to the raw AXO. 


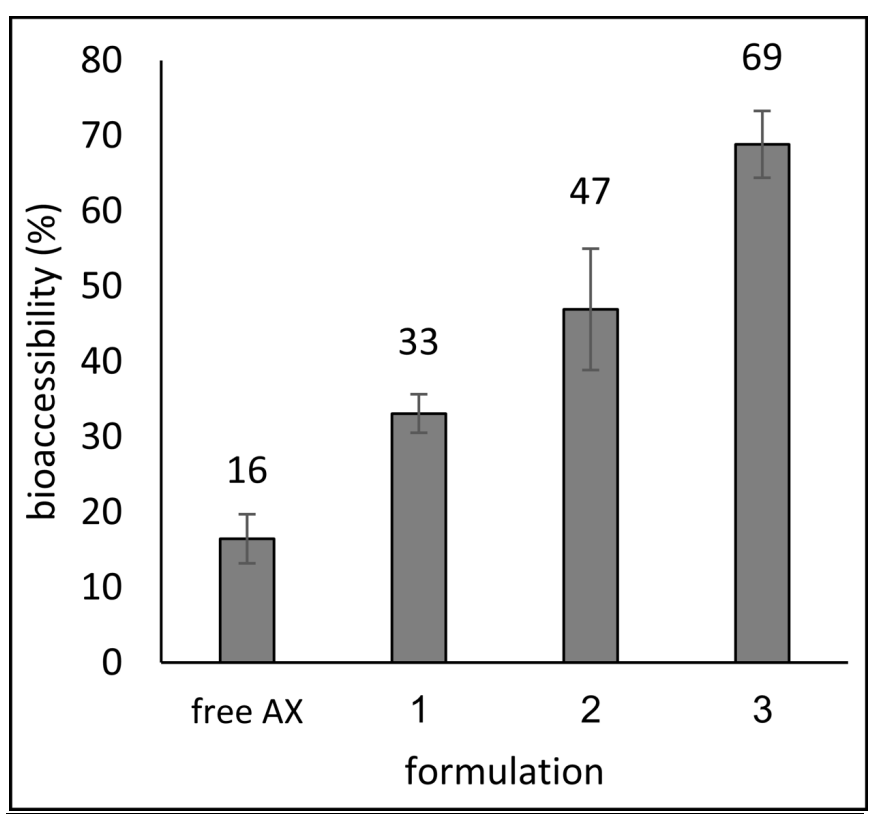

Figure 4 - AX in vitro bioaccessibility in different formulations: unencapsulated AXO in buffer, formulation 1 - AX-PP NPS at 1:1 molar ratio (AX:PP), Formulation 2 - AX+LEC-PP NPs at 2:2:1 molar ratio (AX:LEC:PP), and Formulation 3 $A X+O O-P P$ emulsion at 1\%:3\%:4\% (AXO:OO:PP, \%w/v ratio), after 4 passes through the high-pressure homogenizer. The error bars represent $S E . A X=$ astaxanthin; $A X O=A X$ oleoresin; $L E C=$ lecithin; $N P S=$ nanoparticles; $O O=$ olive oil; $P P$ = potato protein.

\section{Scale-up}

To produce the final emulsion for the bioavailability study, which would be reproducible at a larger scale for future development, several minor changes in the formulation and the equipment were made, as described above. In vitro bioaccessibility was evaluated for each formulation. All formulations were manufactured in an industrial manufacturing environment, using a high-pressure homogenizer (2 passes).

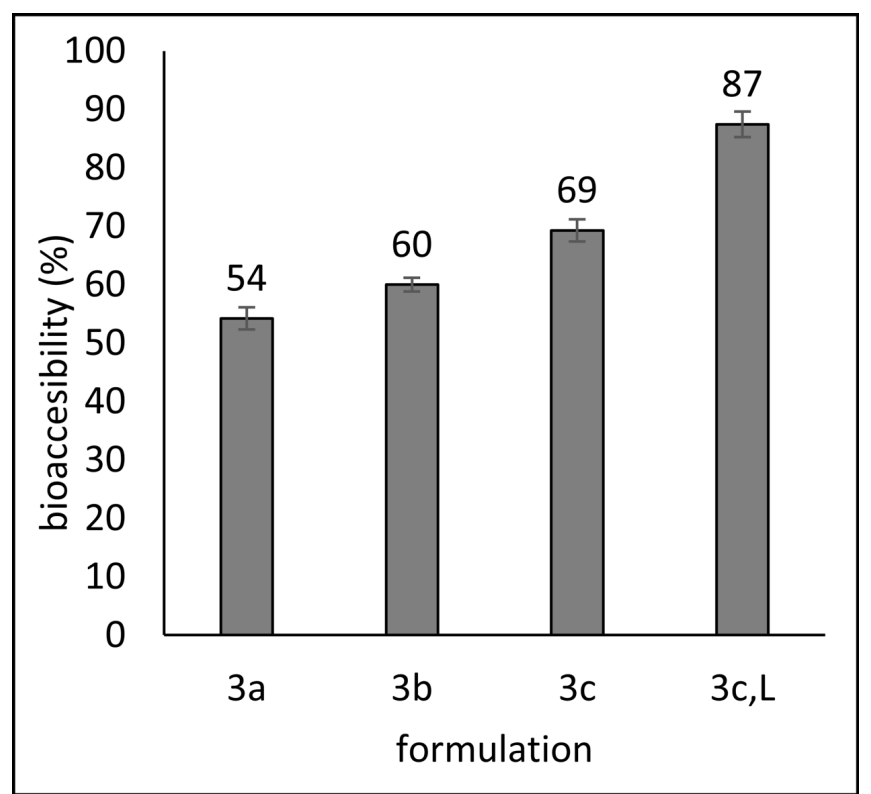

Figure $5-A X$ in vitro bioaccessibility for different emulsion compositions - Formulation $3 a-1 \%: 3 \%: 4 \%$ (AXO:OO:PP, \%w/v ratio), Formulation $3 b-1 \%: 2 \%: 3 \%$ (AXO:OO:PP, \%w/v ratio), Formulation 3c - 1\%:2\%:3\% (AXO:OO:PP, $\%$ w $/ v$ ratio) $+0.15 \%$ maltodextrin, Formulation $3 c, L-$ same as formulation $3 c$, only produced at a larger scale for the clinical study. The error bars represent $S E$. 
Formulation 3a was the same as Formulation 3 (1\%:3\%:4\% (AXO:OO:PP, \%w/v)), and the AX bioaccessibility (Figure 5) was found to be $(54 \pm 3) \%$. The bioaccessibility was calculated as a percentage of the known AX concentration in the original sample.

Formulation $3 \mathrm{~b}$ contained less $\mathrm{OO}$ and protein to obtain a higher percentage of $\mathrm{AX}$ in the final powder (1\%:2\%:3\% (AXO:OO:PP w/w/w)), and the bioaccessibility was found to be $(60 \pm 1) \%$. Formulation $3 \mathrm{c}$ was the same as Formulation $3 \mathrm{~b}$, except for the addition of $0.15 \%$ maltodextrin, to protect the protein during freezing and drying, and the bioaccessibility was found to be $(69 \pm 2) \%$. Formulation $3 \mathrm{c}, \mathrm{L}-$ which was the same as Formulation 3c, only produced at a larger scale for the clinical study, showed the highest bioaccessibility: $(87 \pm 1) \%$.

\section{In vivo bioavailability study in human subjects}

To evaluate the effect of the encapsulation of $\mathrm{AX}$ using $\mathrm{OO}$ and PP, Formulation 3c, which showed the highest in vitro bioaccessibility, was tested in humans in a crossover study to assess its relative oral bioavailability compared to the raw AXO. Thirteen volunteers, who had passed screening, completed the study. Plasma AX concentrations (in $\mu \mathrm{g} / \mathrm{L}$ ) were calculated according to a calibration curve. Figure 6 shows the plasma levels of AX with time after capsules ingestion, for each of the two treatments (median values for the 13 volunteers).

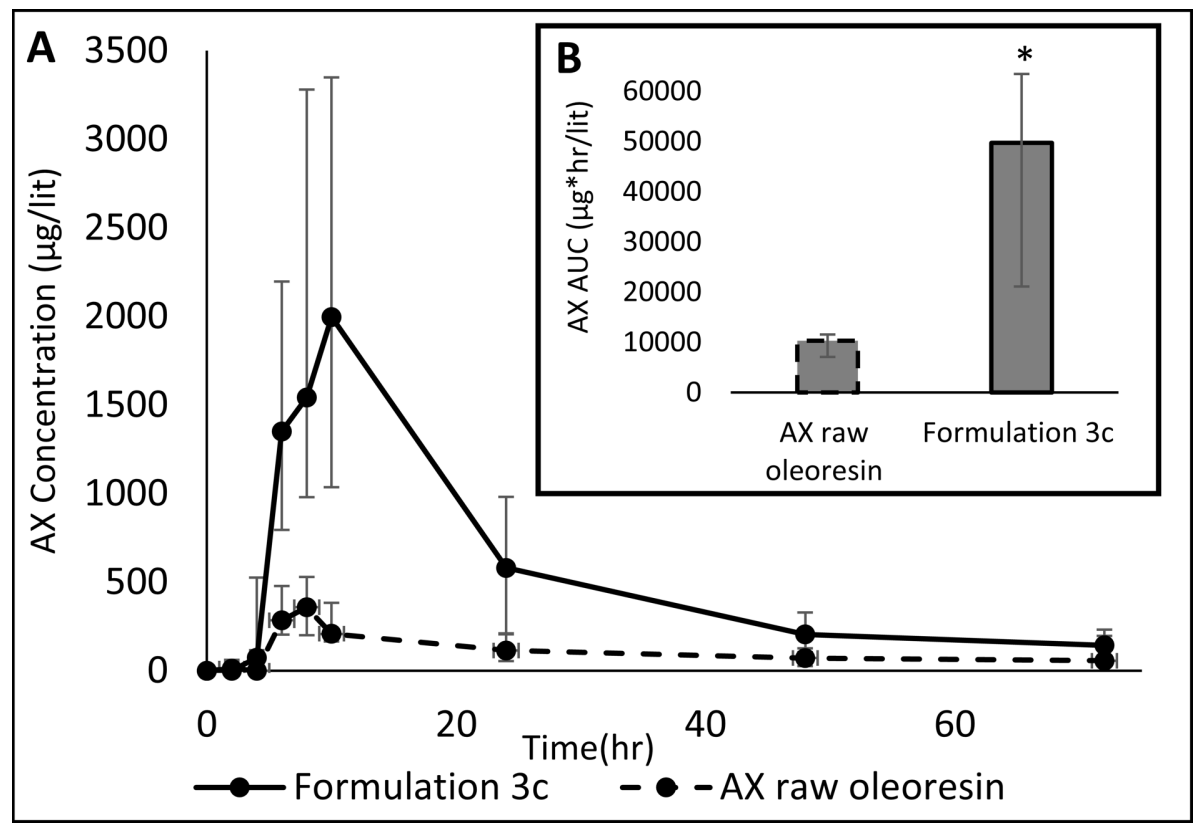

Figure 6 - A. Plasma AX concentration vs. time curves after oral intake of capsules of Formulation 3c (1\%:2\%:3\% (AXO:OO:PP, \%w/v ratio) $+0.15 \%$ maltodextrin) and raw $A X O$ in 13 subjects (Medians and interquartile ranges for each time point are plotted). B. Median AX area under the curve (AUC) and interquartile range of both formulations are plotted. $* P=0.0007$ for the difference between the formulations. $A X=$ astaxanthin; $A X O=A X$ oleoresin; LEC $=$ lecithin; NPs $=$ nanoparticles; $O O=$ olive oil; $P P=$ potato protein.

Table 1 shows the pharmacokinetic parameters for Formulation $3 \mathrm{c}$ and raw AXO. For Formulation $3 \mathrm{c}$, the oral bioavailability of AX was dramatically enhanced compared to that of the untreated AXO: The median AUC and $\mathrm{C}_{\max }$ of Formulation $3 \mathrm{c}$ were 4.8 -fold $(\mathrm{P}<0.001)$ and 6.8 -fold $(\mathrm{P}=0.0012)$ higher than that of the AXO.
All three factors (treatment, time, and their interaction) were significant (Treatment: $\mathrm{P}<0.0001$; time: $<0.0001$; Treatment*time $0.0214)$. The overall treatment effect confirms the differences between microencapsulated AX and the control treatment over the entire timecourse. The treatment $\mathrm{x}$ time interaction indicates significant differences between treatment time-courses. 
Table 1 - Pharmacokinetic parameters obtained in the clinical study following the ingestion of pullulan capsules containing raw $\mathrm{AXO}$ vs. in AX-OO-PP emulsion powder (Formulation 3c,L).

\begin{tabular}{|c|c|c|}
\hline Formulation & Raw AXO & Formulation 3c, $\mathbf{L}$ \\
\hline $\begin{array}{c}\text { Subjects } \\
(\mathbf{n})\end{array}$ & 13 & 13 \\
\hline $\begin{array}{c}\mathbf{A U C} \\
(\boldsymbol{\mu g} * \mathbf{h r} / \mathbf{L})\end{array}$ & $10348(7094-11588)$ & $49724(21156-63445)^{*}$ \\
\hline $\begin{array}{c}\mathbf{C}_{\mathbf{m a x}} \\
(\boldsymbol{\mu g} / \mathbf{L})\end{array}$ & $399(282-865)$ & $2711(1324-4050)^{* *}$ \\
\hline $\begin{array}{c}\mathbf{T}_{\mathbf{1} / \mathbf{2}} \\
(\mathbf{h r})\end{array}$ & $12.4(8.3-20.6)$ & $14.8(10.5-16.2)$ \\
\hline $\begin{array}{c}\mathbf{T}_{\mathbf{m a x}} \\
(\mathbf{h r})\end{array}$ & $8(6-8)$ & $8(8-10)$ \\
\hline $\begin{array}{c}\mathbf{T}_{\mathbf{l a g}} \\
(\mathbf{h r})\end{array}$ & $4(2-6)$ & $4(2-5)$ \\
\hline
\end{tabular}

$* \mathrm{P}=0.0007, * * \mathrm{P}=0.0012$ for comparisons between the two formulations. $\mathrm{AX}=$ astaxanthin; $\mathrm{AXO}=\mathrm{AX}$ oleoresin; $\mathrm{OO}=$ olive oil; $\mathrm{PP}=$ potato protein. Data are presented as medians (25th-75th percentile).

\section{Discussion}

The absorption of carotenoids in the human body depends on several factors, including the structure of the carotenoids, their concentration, the preceding processing conditions, the medium composition, including lipids and other materials consumed together with the carotenoid [31], [32], and the size and structure of the vehicle (e.g. micro- or nanocapsules) delivering them. Carotenoids absorption in the human body begins by releasing the carotenoids from the food matrix during digestion in the stomach and upper small intestine and creating crude lipid emulsion containing other dietary lipids and oils. In the small intestine, the carotenoids from the crude emulsion are incorporated into the mixed micelles. The mixed micelles are heterogeneous micellar aggregates, composed of bile salts excreted from the gall bladder, fatty acids, di- and monoglycerides from digested lipids, phospholipids, and other lipid soluble compounds such as carotenoids. The presence of fat in the meal stimulates the secretion of bile salts and lipases. It provides phospholipids, glycerides, and free fatty acid for the formation of the mixed micelles, and therefore it generally im- proves carotenoid absorption. The mixed micelles are passed diffusively through the unstirred water layer of the intestinal mucosa, before being transported across the epithelial cell membranes [33], [34]. Carotenoids were considered to penetrate the enterocytes by passive diffusion, but recent studies show that it also occurs by facilitated (active) uptake. Different transporters on the enterocyte membrane take part in carotenoid transport, such as SR-B1, CD36, and NPC1L1 [35]. After being transported into the enterocytes, carotenoids are incorporated into chylomicrons by the Golgi apparatus, and secreted into the lymph fluid. From the lymphatic system, the chylomicrons are transported via the thoracic duct, then the subclavian vein into the entire blood circulation, and then the liver [34].

Oral bioavailability normally refers to the fraction of the component that enters the bloodstream. The bioavailability of bioactive compounds, such as AX, can be increased by decreasing particle size (e.g., by using low molecular weight surfactants, like lecithin, or macromolecular surfactants like amphiphilic proteins, e.g., PP), or by combining them with lipids 
[27], [36]. In this study, we compared these approaches by creating AX NPs with PP to decrease particle size, with or without the combi- nation of phospholipids, or forming AX emulsion with PP and OO. Figure 7 describes the proposed model structures of the three systems studied.

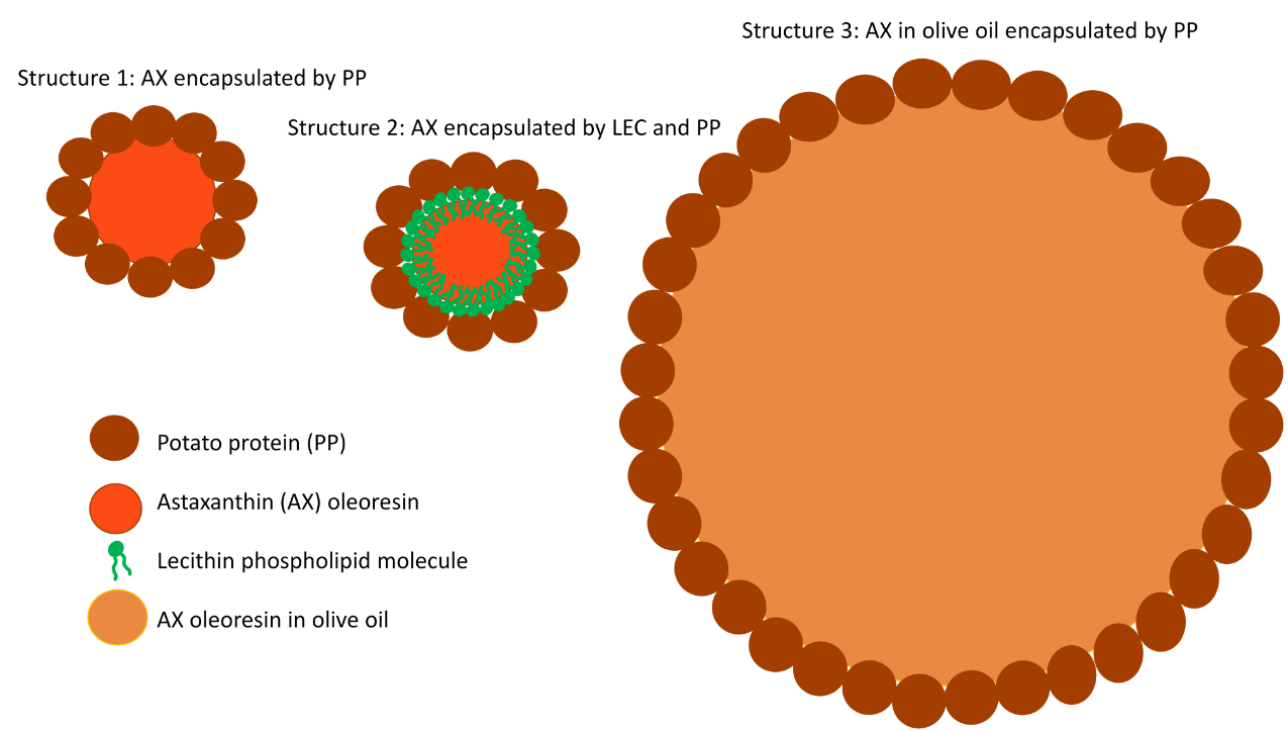

Figure 7 - Proposed model structures of the three systems studied herein. $A X=$ astaxanthin; $A X O=A X$ oleoresin; LEC $=$ lecithin; $P P=$ potato protein.

All those systems showed improvement of AX in vitro bioaccessibility compared to that of unencapsulated AXO particles. Encapsulating $\mathrm{AX}$ in a PP-OO emulsion showed the greatest improvement in bioaccessibility, although the average particle diameter of this system was the highest of all delivery systems studied. A previous study described the encapsulation of AXcontaining lipid extract from shrimp waste by spray drying and different wall materials. In all encapsulated AX formulations, there was an increase in AX bioaccessibility, compared to nonencapsulated lipid extract. Those findings were related to the high solubility of the microparticles formed [37]. In another study, AX nanoemulsions were made using sodium caseinate and different oils. Those emulsions improved the bioaccessibility of AX, with the most significant improvement achieved by adding $\mathrm{OO}$ (68.4\%), close to the bioaccessibility of Formulation 3 in the current study (69\%). In formulation 3, the particle size was much larger than in the nano-emulsions, which indicated that combining AX with OO was more effective than decreasing particle size. While the use of an easily digestible protein shell, which is hydrolyzed in the stomach and small intestine [38] helps improving bioaccessibility, the higher bioaccessibility of the oil-containing microparticles can be attributed to the ability of the oil to induce the formation of mixed micelles that could solubilize the hydrophobic carotenoids [39].

Scale up of the manufacturing process was performed to prepare sufficient amounts needed for the clinical study. In this process, the same formulation was prepared in larger volumes using pilot-scale equipment in an industrial factory. Moreover, slight changes in the formulation were made to increase the AX concentration in the final formulation. When considering Formulation 3, versus 3a, the bioaccessibility decreased when moving from lab scale to a larger scale (from $69 \%$ to $54 \%$ ), apparently mainly due to the change in equipment. In the other formulations tested, the proportion of AX, compared to $\mathrm{OO}$ and $\mathrm{PP}$, was raised to decrease the final powder volume per active material. Furthermore, the addition of maltodextrin as a cryoprotectant and reconstitutability enhancer was tested. Interestingly, the decrease in the proportion of $\mathrm{OO}$ and $\mathrm{PP}$ to $\mathrm{AX}$ improved $\mathrm{AX}$ bioaccessibility (from 54 to $60 \%$ ), and the addition of maltodextrin further increased the bioaccessibility to $69 \%$, probably by decreasing aggregation upon freeze-drying. The large batch prepared for the clinical study showed the highest bioaccessibility of all formulations 
tested, $87 \%$. This result was an unexpected positive effect of the scale up, which may be due to the slower freezing and drying, resulting from the larger mass and equipment used. Further study will be required to explain this interesting observation.

The final emulsion composition used in the clinical study was 1\%:2\%:3\% (AXO:OO:PP, $\mathrm{w} / \mathrm{w} / \mathrm{w})+0.15 \%$ maltodextrin. In the randomized, double-blind crossover study, each of the 13 healthy volunteers received $15 \mathrm{mg}$ of $\mathrm{AX}$, once as raw AXO and once in the formulation developed, with a one-week washout period in between. The AX AUC and $\mathrm{C}_{\max }$ for capsules containing Formulation $3 \mathrm{c}, \mathrm{L}$ were 4.8 -fold higher than those for capsules containing raw AXO, a highly significant difference, despite the small number of study participants $(\mathrm{P} \leq 0.001)$. These results corresponded to the findings of the preceding in vitro bioaccessibility studies, indicating that dissolving $\mathrm{AX}$ in $\mathrm{OO}$ and encapsulating it with PP significantly improves oral bioavailability of AX. In another study, a single dose of $40 \mathrm{mg}$ of AX, as lipidbased formulations, was tested in a clinical study, comparing different lipids to AX alone. The 3 lipid-based formulations studied ([A] saturated long-chain triglyceride (palm oil) with polysorbate 80 (Tween 80 , a synthetic surfactant); [B] glycerol mono- and dioleate and Tween 80; and [C] glycerol mono- and dioleate, Tween 80 and sorbitan monooleate], all showed increased bioavailability, from 1.7 to 3.7 times that of the reference formulation (consisting algal meal and dextrin in hard gelatin capsules). The highest oral bioavailability was reported for Formulation B, containing a high content of Tween 80 [27]. Hence, our study is in accord with studies showing lipids may improve AX bioavailability, but we have demonstrated a highly significant bioavailability improvement using only natural non-allergenic plant-based ingredients.

\section{Conclusions}

To conclude, the final formulation developed, based on potato protein and olive oil $(1: 2: 3 \mathrm{w} / \mathrm{w} / \mathrm{w}$ AXO:OO:PP), with $0.15 \%$ maltodextrin, produced using pilot-scale industrial equipment, exhibited a 4.8-fold higher systemic astaxanthin concentration in human subjects compared to the raw AXO formulation. Thus, this non-allergenic, vegan, PP-based delivery system made of "All-Natural Ingredients" offers great promise for increasing oral bioavailability of lipophilic bioactives, in particular for the development of dietary supplements of astaxanthin and other carotenoids or oil-soluble bioactives.

\section{Acknowledgements}

This study was kindly supported by the Nofar Program of the Israeli Innovation Authority (Grant Number 59436) and by Frutarom Industries Ltd. We thank Dr. Yoni Glickman (Frutarom) who raised the need for this study, Yehudit Chen-Zion, Dr. Fabiana Bar-Yoseph, Gai Ben Dror, Dr. Yael Richter (Enzymotech/Frutarom) for their help in sample production and preparations for the clinical study, and we also thank Prof. Naim Shehadeh, Dr. Elena Segal, Tzipi Lazarovich, Margalit Levi, Amira Halabi, Dolly Haddad, Dr. Mariel Kaplan, Dr. Ronit Tamir, and Anna Ziskind, from the Rambam Health Care Campus for their help in performing the clinical study; we thank Shira Engelberg, for her help with several experiments, Boris Herscovici, Lior Harpaz, and Dikla Gina for administrative support, and Dr. Elliot Sprecher for the statistical analysis. A special thanks to Prof. Yoav Sharoni, from Ben Gurion University, Beer Sheva, Israel, for very helpful advice along the study.

\section{Conflict of Interest Statement}

Authors declare that they have no conflict of interest related to this paper. For a signed statement, please contact the journal office: editor@precisionnanomedicine.com

Quote this paper as: Abuhassira-Cohen Y, Edelman R, Abbas R, Kurnik D, Shibel R, and Livney YD, Enhancing the oral bioavailability of natural astaxanthin using plant-based micro- and nano-encapsulation materials: Results of an in vitro evaluation and a crossover study in humans, Precis. Nanomed. 2020;3(3):641-655. https://doi.org/10.33218/001c.16781 


\section{References}

[1] R. R. Ambati, S. M. Phang, S. Ravi, and R. G. Aswathanarayana, "Astaxanthin: sources, extraction, stability, biological activities and its commercial applications--a review," Mar Drugs, vol. 12, no. 1, pp. 128$152,2014$.

[2] S. F. Lockwood, S. O'Malley, and G. L. Mosher, "Improved aqueous solubility of crystalline astaxanthin (3,3'-dihydroxy- $\beta, \beta$-carotene-4,4'-dione) by Captisol ${ }^{\circledR}$ (sulfobutyl ether $\beta$-cyclodextrin)," J. Pharm. Sci., vol. 92, no. 4, pp. 922-926, 2003.

[3] Q. Shen and S. Y. Quek, "Microencapsulation of astaxanthin with blends of milk protein and fiber by spray drying," J. Food Eng., vol. 123, pp. 165-171, 2014.

[4] Vazhiyil Venugopal, "Marine Products for Healthcare: Functional and Bioactive Nutraceutical," CRC Press New York, 2008, p. 321-343.

[5] Y. M. a Naguib, "Antioxidant activities of astaxanthin and related carotenoids," J. Agric. Food Chem., vol. 48, no. 4, pp. 1150-1154, 2000.

[6] S. Fakhri, F. Abbaszadeh, L. Dargahi, and M. Jorjani, "Astaxanthin: A mechanistic review on its biological activities and health benefits," Pharmacol. Res., vol. 136, no. August, pp. 1-20, 2018.

[7] S. Davinelli, M. E. Nielsen, and G. Scapagnini, "Astaxanthin in skin health, repair, and disease: A comprehensive review," Nutrients, vol. 10, no. 4, pp. 1-12, 2018.

[8] E. Nowak, Y. D. Livney, Z. Niu, and H. Singh, "Delivery of bioactives in food for optimal efficacy: What inspirations and insights can be gained from pharmaceutics?," Trends Food Sci. Technol., vol. 91, no. June, pp. 557-573, 2019.

[9] PubChem, "Astaxanthin." [Online]. Available: https://pubchem.ncbi.nlm.nih.gov/compound/Astaxanthin\#section=Color-For. [Accessed: 13-Aug-2020].

[10] G. Israeli-Lev, M. Pitchkhadze, S. Nevo, L. Fahoum, E. Meyron-Holtz, and Y. D. Livney, "Harnessing proteins to control crystal size and morphology, for improved delivery performance of hydrophobic bioactives, using genistein as a model," Food Hydrocoll., vol. 63, pp. 97-107, 2017.

[11] M. M. R. M. M. Affandi, T. Julianto, and A. B. A. Majeed, "Enhanced Oral Bioavailability of Astaxanthin with Droplet Size Reduction," Food Sci. Technol. Res., vol. 18, no. 4, pp. 549-554, 2012.

[12] S. David and Y. D. Livney, "Potato protein based nanovehicles for health promoting hydrophobic bioactives in clear beverages," Food Hydrocoll., vol. 57, pp. 229-235, Jun. 2016.

[13] A. C. Ross et al., "The 2011 Report on Dietary Reference Intakes for Calcium and Vitamin D from the Institute of Medicine: What Clinicians Need to Know," J. Clin. Endocrinol. Metab., vol. 96, no. 1, pp. $53-58,2011$.

[14] M. C. Ralet and J. Guéguen, "Fractionation of Potato Proteins: Solubility, Thermal Coagulation and Emulsifying Properties," LWT - Food Sci. Technol., vol. 33, no. 5, pp. 380-387, 2000.

[15] P. N. Ezhilarasi, P. Karthik, N. Chhanwal, and C. Anandharamakrishnan, "Nanoencapsulation Techniques for Food Bioactive Components: A Review," Food Bioprocess Technol., vol. 6, no. 3, pp. 628647, 2013.

[16] K. Pan, Y. Luo, Y. Gan, S. J. Baek, and Q. Zhong, "pH-driven encapsulation of curcumin in selfassembled casein nanoparticles for enhanced dispersibility and bioactivity," Soft Matter, vol. 10, no. 35, p. 6820, 2014.

[17] M. Haham et al., "Stability and bioavailability of vitamin D nanoencapsulated in casein micelles," Food Funct., vol. 3, no. 7, p. 737, Jun. 2012.

[18] E. Semo, E. Kesselman, D. Danino, and Y. D. Livney, "Casein micelle as a natural nano-capsular vehicle for nutraceuticals,” FOOD Hydrocoll., vol. 21, pp. 936-942, 2007.

[19] S. David, Y. Zagury, and Y. D. Livney, "Soy $\beta$-Conglycinin-Curcumin Nanocomplexes for Enrichment of Clear Beverages," Food Biophys., vol. 10, no. 2, pp. 195-206, Jun. 2015.

[20] Gilad Markman and Yoav D. Livney, "Maillard-conjugate based core-shell co-assemblies for nanoencapsulation of hydrophobic nutraceuticals in clear beverages," Food \& function, 2012. .

[21] Y. Levinson, G. Israeli-Lev, and Y. Y. D. Livney, "Soybean $\beta$-Conglycinin Nanoparticles for delivery of hydrophobic nutraceuticals," Food Biophys., vol. 9, no. 4, pp. 332-340, 2014. 
[22] Y. Cohen, S. Ish-Shalom, E. Segal, O. Nudelman, A. Shpigelman, and Y. D. Livney, "The bioavailability of vitamin D3, a model hydrophobic nutraceutical, in casein micelles, as model protein nanoparticles: Human clinical trial results," J. Funct. Foods, vol. 30, 2017.

[23] Y. Levinson, S. Ish-Shalom, E. Segal, and Y. D. Livney, "Bioavailability, rheology and sensory evaluation of fat-free yogurt enriched with VD3 encapsulated in re-assembled casein micelles," Food Funct., vol. 7, no. 3, 2016.

[24] R. M. Clark, L. Yao, L. She, and H. C. Furr, "A comparison of lycopene and astaxanthin absorption from corn oil and olive oil emulsions," Lipids, vol. 35, no. 7, pp. 803-806, 2000.

[25] J. B. Maltby, L. J. Albright, C. J. Kennedy, and D. a. Higgs, "Effect of route of administration and carrier on bioavailability and kinetics of astaxanthin in Atlantic salmon Salmo salar L.," Aquac. Res., vol. 34, no. 10, pp. 829-838, 2003.

[26] C. Bustos-Garza, J. Yáñez-Fernández, and B. E. Barragán-Huerta, "Thermal and pH stability of spray-dried encapsulated astaxanthin oleoresin from Haematococcus pluvialis using several encapsulation wall materials,” Food Res. Int., vol. 54, no. 1, pp. 641-649, 2013.

[27] J. Mercke Odeberg, Å. Lignell, A. Pettersson, and P. Höglund, "Oral bioavailability of the antioxidant astaxanthin in humans is enhanced by incorporation of lipid based formulations," Eur. J. Pharm. Sci., vol. 19, no. 4, pp. 299-304, 2003.

[28] M. Minekus et al., "A standardised static in vitro digestion method suitable for food - an international consensus," Food Funct, vol. 5, no. 6, pp. 1113-1124, 2014.

[29] B. B. Osterlie M., M. Østerlie, and B. Bjerkeng, "Plasma appearance and distribution of plasma lipoproteins of men after single dose administration of astaxanthin," J. Nutr. Biochem., vol. 2863, no. 00, pp. 482-490, 2000.

[30] T. Iwamoto, Matsumoto, M. Wataru Kazuaki Hosoda, Miki, Yamamoto, and and K. Kondo, "Inhibition of Low-Density Lipoprotein Oxidation by Astaxanthin,” J. Atheroscler. Thromb., vol. 7, no. 4, pp. 216-222, 2000.

[31] D. J. McClements and H. Xiao, "Excipient foods: designing food matrices that improve the oral bioavailability of pharmaceuticals and nutraceuticals," Food Funct, vol. 5, no. 7, pp. 1320-1333, 2014.

[32] L. Salvia-Trujillo, C. Qian, O. Martín-Belloso, and D. J. McClements, "Influence of particle size on lipid digestion and $\beta$-carotene bioaccessibility in emulsions and nano-emulsions," Food Chem., vol. 141, no. 2, pp. 1475-1480, 2013.

[33] S. R. Goltz and M. G. Ferruzzi, "Carotenoid Bioavailability: Influence of Dietary Lipid and Fiber," in Carotenoids and Human Health, 2013, pp. 111-128.

[34] A. P. Neilson, K. M. Goodrich, and M. G. Ferruzzi, "Bioavailability and metabolism of bioactive compounds from foods," in Nutrition in the Prevention and Treatment of Disease, Fourth Edi., Elsevier Inc., 2017, pp. 301-319.

[35] E. Reboul, "Mechanisms of Carotenoid Intestinal Absorption: Where Do We Stand?," Nutrients, vol. 11, pp. 1-12, 2019.

[36] E. Acosta, "Bioavailability of nanoparticles in nutrient and nutraceutical delivery," Curr. Opin. Colloid Interface Sci., vol. 14, no. 1, pp. 3-15, 2009.

[37] P. Montero, M. M. Calvo, M. C. Gómez-Guillén, and J. Gómez-Estaca, "Microcapsules containing astaxanthin from shrimp waste as potential food coloring and functional ingredient: Characterization, stability, and bioaccessibility," LWT - Food Sci. Technol., vol. 70, pp. 229-236, Jul. 2016.

[38] R. Edelman, S. Engelberg, L. Fahoum, E. G. Meyron-Holtz, and Y. D. Livney, "Potato proteinbased carriers for enhancing bioavailability of astaxanthin," Food Hydrocoll., vol. 96, pp. 72-80, Nov. 2019.

[39] X. Liu et al., "Nanoemulsion-Based Delivery Systems for Nutraceuticals: Influence of Long-Chain Triglyceride (LCT) Type on In Vitro Digestion and Astaxanthin Bioaccessibility," Food Biophys., vol. 13, no. 4, pp. 412-421, 2018. 